Original Article

\title{
Femoral venous flow velocity during passive ankle exercise in patients with chronic spinal cord injury
}

\author{
Tomoaki Hayakawa, PT, MA"), Motoyuki Abe, MD, $\mathrm{PhD}^{2)^{*}}$ \\ 1) Aozora Zaitaku Clinic, Japan \\ 2) Faculty of Care and Rehabilitation, Seijoh University: 2-172 Fukinodai, Tokai-shi, Aichi 476-8588, Japan
}

\begin{abstract}
Purpose] We examined the effect of passive ankle exercise on femoral venous flow in paralyzed, atrophied lower legs of patients with chronic spinal cord injury. [Participants and Methods] Ten males with complete spinal cord injury at the T6-12 levels and ten able-bodied males participated in this study. B-mode muscle ultrasound was performed on the medial gastrocnemius muscle to evaluate muscle atrophy. Doppler ultrasound was used to measure the time-averaged mean velocity in the femoral vein at rest and during passive motion of the ankle. [Results] Mean muscle thickness was significantly reduced in the spinal cord injury group, showing a mean value of $67.2 \%$ compared with able-bodied participants. No significant differences were observed in the time-averaged mean velocity at rest between the groups, although they were slightly lower in the spinal cord injury group than in the able-bodied group. During passive motion, no significant difference was observed in the time-averaged mean velocity between the groups, although the mean value in those with spinal cord injury was $65.9 \%$ that of the able-bodied group. Time-averaged mean velocity increased in both groups, compared with baseline. [Conclusion] Passive ankle exercise increased time-averaged mean velocity in spinal cord injury, but venous blood velocity was reduced in spinal cord injury compared to the able-bodied group. We believe that significant muscle atrophy affected our results in chronic spinal cord injury.

Key words: Paraplegia, Passive ankle exercise, Venous flow velocity
\end{abstract}

(This article was submitted Mar. 14, 2019, and was accepted May 30, 2019)

\section{INTRODUCTION}

Deep venous thrombosis (DVT) is an important cause of morbidity and mortality in patients with spinal cord injury (SCI), often due to fatal pulmonary embolism. DVT occurs in $47-72 \%$ of these patients in the first 2 weeks after injury ${ }^{1,2)}$. In Japan, $21 \%$ of patients with acute cervical cord injury developed DVT, with most cases being asymptomatic ${ }^{3}$.

Despite increased awareness of DVT in acute SCI, little attention has been paid to the occurrence of DVT in patients with chronic SCI long after sustaining a SCI. Although patients with acute SCI have benefited from various treatments, DVT prophylaxis is not usually provided to patients with chronic SCI, even though patients with both acute and chronic SCI present with the same Virchow's triad conditions (stasis, hypercoagulability, and vessel intimal injury) ${ }^{4}$. However, the prevalence of DVT is lower in chronic SCI than that observed in acute $\mathrm{SCI}^{5)}$.

Although several pharmaceutical prophylactic approaches are available to prevent DVT, mechanical methods are still commonly used to increase venous flow velocity. These include compression stockings, intermittent pneumatic pressure, deep breathing, active ankle exercises. The use of compression stockings is common in the context of various diseases, although the CLOT1 study reported that their use did not prevent DVT following acute stroke ${ }^{6}$.

*Corresponding author. Motoyuki Abe (E-mail: abe-m@seijoh-u.ac.jp)

(C2019 The Society of Physical Therapy Science. Published by IPEC Inc.

(c) (i) $\odot$ This is an open-access article distributed under the terms of the Creative Commons Attribution Non-Commercial No Deriva-

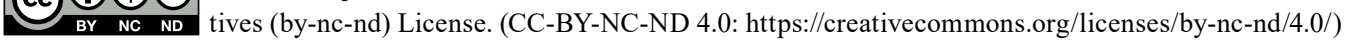


Active ankle exercise has been reported as the most effective mechanical method that can improve venous velocity and avoid venous stasis ${ }^{7,8)}$. Venous flow velocity in the femoral vein was 1.20-2.04 times higher than the resting value in ablebodied individuals $(\mathrm{AB})$ with active ankle exercise ${ }^{8,9)}$. Active leg exercise promotes the skeletal muscle pump and enhances venous return to the heart, but patients with SCI cannot move actively their own paralyzed legs.

On the other hand, few studies have focused on the changes of venous return by passive ankle exercise. Sochart et al. reported that both passive and active ankle exercise resulted in an increase in mean blood velocities in the femoral vein over the resting levels in $\mathrm{AB}$, while the active exercises produced greater changes ${ }^{9}$. However, no study has reported the change in venous flow velocity during passive ankle exercise in patients with chronic SCI, so the effect remains unclear in this population compared with $\mathrm{AB}$. In addition, chronic SCI is expected to lead to muscle atrophy for a long duration after injury. In addition, the relationship between muscle atrophy and venous flow velocity during passive ankle exercise remains unclear. The aim of this study was to investigate the effect of passive ankle exercise on femoral venous velocity of the paralyzed leg with muscle atrophy in patients with chronic SCI. This study may provide a base for reducing the risk of DVT in SCI.

\section{PARTICIPANTS AND METHODS}

Ten paraplegic males with post-traumatic SCI, all of whom were at least 1 year post-injury, were recruited to this study. All had complete spinal cord lesions between the T6 through T12 segments (American Spinal Injury Association Grade A). All were independently using manual wheelchairs. As controls, ten AB males with matched age and physique were included in the study.

All patients with chronic SCI and AB underwent a pre-participation medical examination, a thorough review of their medical history including cardiac and pulmonary conditions, and a neurological assessment. None of the participants presented with any cardiovascular, pulmonary, or metabolic disease at the time of assessment, and none were taking any medications that were likely to interfere with the cardiovascular system or affect the study results. Individuals with a history of DVT of the lower extremities were excluded from the study. The anthropometric and neurological characteristics of the participants are shown in Table 1. No significant differences in age, height, or weight were present between the 2 groups.

Written informed consent was obtained from all participants, and the protocol was approved by the Seijoh University Research Ethics Committee (No. 2012C0003).

Although none of the participants had a history of DVT, all first underwent a venous duplex ultrasound examination of the lower extremities to identify any individual with an asymptomatic DVT prior to this study.

A B-mode muscle ultrasound using a 12-MHz linear array transducer was performed on the left medial gastrocnemius muscle in the upper region of the lower leg to evaluate for muscle atrophy. The ultrasound was obtained from the upper $3 / 8$ th of the lower thigh, $2 \mathrm{~cm}$ from the longitudinal central line. The muscle thickness was defined as the distance within the internal fascia.

The passive ankle exercise involved passive plantarflexion and dorsiflexion in the sitting position on a fixed wheelchair in preparation for this study. Using a metronome to pace the rate of passive flexion, a single examiner (TH) performed the exercise with the participant's ankle once per second for $20 \mathrm{~s}$, reaching the distance of maximum joint arc. All measurements were performed in a temperature-controlled room $\left(21-24^{\circ} \mathrm{C}\right)$.

To measure venous flow velocity, the measurement point of the probe was placed over the femoral vein below the inguinal ligament. Pulsed wave Doppler ultrasound with an $8-\mathrm{MHz}$ linear array transducer was conducted. We measured the time averaged maximum flow velocity (TAMV) $(\mathrm{cm} / \mathrm{s})$ of the left femoral vein at both rest and during passive ankle exercise. Measurements were taken with the participant breathing normally in a resting seated position on a fixed wheelchair. These measurements of angle-corrected flow velocities were taken with the sample volume covering over $2 / 3$ of the entire vessel diameter, making the corrections for the angle of insonation under 60 degrees.

Table 1. Descriptive characteristics of the study participants

\begin{tabular}{lcc}
\hline & $\mathrm{SCI}(\mathrm{n}=10)$ & $\mathrm{AB}(\mathrm{n}=10)$ \\
\hline Age (years) & $34.6 \pm 4.7$ & $32.0 \pm 6.5$ \\
Height $(\mathrm{cm})$ & $171.5 \pm 6.8$ & $174.9 \pm 4.0$ \\
Weight $(\mathrm{kg})$ & $67.5 \pm 12.8$ & $67.7 \pm 9.9$ \\
Level of injury & $\mathrm{T} 6-\mathrm{T} 12$ & - \\
Duration from injury (months) & $112.1 \pm 90.7$ & - \\
Swelling of leg (Yes/No) & $0 / 10$ & $0 / 10$ \\
Use of anticoagulant (Yes/No) & $0 / 10$ & $0 / 10$ \\
\hline
\end{tabular}

All values are expressed as mean $\pm \mathrm{SD}$.

SCI: spinal cord injury; AB: able-bodied; T: thoracic.

These parameters did not differ significantly between groups.

All cases of paraplegia were complete (American Spinal Injury Association; ASIA:A) spinal cord lesions. 
The venous diameter of the femoral vein $(\mathrm{mm})$ was measured as the distance within the internal lumen at the time of rest, simultaneously at the same site as described above, using B-mode ultrasound.

An ultrasound diagnostic imaging device (Xario; Toshiba Medicals Co., Ltd., Tochigi, Japan) was used by the same examiner (MA) to detect asymptomatic DVTs and to measure the muscle thickness, the TAMV, and the venous diameter as mentioned above.

All statistical analyses were conducted using SPSS 17 software (SPSS Inc., Chicago, IL, USA). An unpaired t-test was used to determine the statistical differences between the groups with regard to the participant physical characteristics, the muscle thickness, the TAMV, and the venous diameter. A paired t-test was used to analyze the effect of passive ankle exercise on the TAMV. Statistical significance was set at $\mathrm{p}<0.05$.

\section{RESULTS}

All chronic SCI participants presented with asymptomatic DVT in the femoral, popliteal, or soleus veins diagnosed by duplex ultrasound. The study could continue without an added risk of pulmonary embolism because all detected emboli were completely organized and were unlikely to become dislodged.

Mean medial gastrocnemius muscle thickness was significantly lower in chronic SCI participants (unpaired t-test, $\mathrm{p}<0.005)$. All patients with chronic SCI had more hyperechoic muscles on B-mode ultrasound than AB.

The TAMV at rest and during passive ankle exercise while sitting in a wheelchair are shown in Table 2. No significant differences were observed in TAMV at rest and during passive ankle exercise between the groups, although the values were slightly lower in chronic SCI than in AB.

TAMV during passive ankle exercise significantly increased in SCI and AB compared with the resting values ( $\mathrm{p}=0.001$, $\mathrm{p}=0.004$, respectively). In patients with SCI and in AB, TAMV values during passive ankle exercise were 2.39 and 2.74 times higher, respectively, than the resting values. No significant difference was present in venous diameter, although SCI was slightly lower in chronic SCI than in AB, as with the result for muscle thickness.

\section{DISCUSSION}

This study investigated the prevalence of DVT, the muscle thickness of the lower limb, the TAMV during passive ankle exercise, and the venous diameter of patients with chronic paraplegia. Our study had 3 important findings, as described below.

First, it was found that all chronic SCI patients who were more than 1 year out from their injury had asymptomatic DVTs, but these were considered secure since all detected emboli were completely organized. This high incidence of DVT has not been reported previously, and highlights the need for investigation of DVT in patients with chronic SCI. Lamb et al. reported that the incidence of symptomatic DVT in SCI decreased after 6 months but asymptomatic DVTs were still present in small percentage of patients (overall $10 \%)^{10}$ ). Thus, more attention should be paid to the possibility of DVT in the context of chronic SCI, even when patients have no symptoms.

Second, it was found that significant leg muscle atrophy may be linked to a degeneration of the leg in patients with chronic SCI with lower thoracic injuries, with muscle thickness being $67.2 \%$ that of AB in those chronic SCI. McCully et al. reported that the muscle fat content was 6 times higher in patients with chronic SCI than in AB using magnetic resonance imaging ${ }^{11)}$. This may reflect muscle hyperechoic changes of the lower leg by ultrasound, although $\mathrm{AB}$ had hypoechoic changes that were different from those with SCI.

Third, it was found that TAMV during passive ankle exercise significantly increased even in SCI, but those with SCI had slightly lower values compared with $\mathrm{AB}$, similar to the results for muscle thickness. Our results showed that the skeletal muscle pump of the calf muscles plays an important role in increasing venous return during passive exercise even in patients with chronic SCI. Those with chronic SCI had a smaller ratio of TAMV at baseline compared with AB (75.0\% that of AB). These values were similar to the ratio of muscle thickness of those with SCI compared with $\mathrm{AB}$ (67.2\% that of AB). TAMV during passive ankle exercise increased 2.39 and 2.74 times higher in those with chronic SCI and in AB, respectively,

Table 2. Muscle thickness, TAMV, and venous diameter in SCI and AB

\begin{tabular}{llccc}
\hline Parameters & Rest or exercise & SCI $(\mathrm{n}=10)$ & AB $(\mathrm{n}=10)$ & $\mathrm{p}$ value \\
\hline Muscle thickness $(\mathrm{mm})$ & & $9.19 \pm 3.56$ & $13.70 \pm 2.20$ & 0.005 \\
TAMV $(\mathrm{cm} / \mathrm{s})$ & Baseline & $2.41 \pm 1.54$ & $3.20 \pm 2.35$ & 0.420 \\
& Passive ankle exercise & $5.76 \pm 2.78$ & $8.77 \pm 6.08$ & 0.190 \\
Venous diameter $(\mathrm{mm})$ & Baseline & $7.59 \pm 2.18$ & $9.76 \pm 2.21$ & 0.052 \\
\hline
\end{tabular}

All values are expressed as mean $\pm \mathrm{SD}$.

SCI: spinal cord injury; AB: able-bodied; TAMV: time-averaged mean velocity.

TAMV during passive ankle exercise were significantly higher in SCI and AB than the baseline $(p=0.001, p=0.004$, respectively). 
compared with resting values in our study, though a similar previous study reported that passive ankle exercise led to values 1.20 times higher in $\mathrm{AB}^{9}$ ). These differences may be because the frequency and strength of the exercise affected the results, as participants in our study carried out more rigorous and frequent exercises. A decrease in venous distensibility and capacity due to muscle atrophy in patients with chronic SCI could explain the differences in TAMV between participants with chronic $\mathrm{SCI}$ and $\mathrm{AB}$ in this study. The effect of passive ankle exercise in SCI was shown to be $65.9 \%$ of the TAMV compared with $\mathrm{AB}$. This may also be due to muscle atrophy of the lower limbs and little blood flow retention of the lower paralyzed leg in SCI. This may indicate an alteration of blood vessel and muscle atrophy associated with sympathetic denervation, and a subsequent decrease in blood distribution to the lower paralyzed leg a long period after injury. We estimated differences in volumes of venous return in both groups based on our data.

Venous diameter was slightly smaller in those with chronic SCI than in AB, but the differences were not statistically significant. Venous diameter and TAMV plays important roles in determining the venous flow volume that returns to the heart. Cross-sectional area of the femoral vein was calculated by the radius of the venous diameter, pi squared. Consequently, it was considered that venous flow volume was smaller in chronic SCI than in AB, approximately half in this study. We described the unconventional state of venous hemodynamics in chronic SCI in order to help alter venous stasis of paralyzed legs.

The most effective mechanical method to improve venous flow velocity is believed to be active ankle exercise ${ }^{7,8)}$, but patients with SCI can perform passive exercise of their own paralyzed legs. Deep breathing is an alternative method that can give rise to increased respiratory pump, increasing venous return to the heart from the legs ${ }^{8}$. Although the benefits of venous return during deep breathing are obvious, excessive and continuous deep breathing may cause hyperventilation syndrome, which can result in dizziness, perceived dyspnea, and other difficulties. Therefore, the use of that method alone is not recommended in patients with SCI. Venous flow velocity does not markedly improve (1.06 times greater than the resting value) with the use of compression stockings in $\mathrm{AB}^{12)}$. It was considered that the use of compression stockings for SCI may be not effective as in the context of stroke ${ }^{6}$.

This study had some limitations. First, because we had a small sample size, the results should be viewed with caution. Second, the level of SCI (T6-T12) may have affected the extent of muscle atrophy of the lower extremities. More participants are needed to determine its effect and relationship. Third, measurements were taken only in the sitting position in this study, and venous hemodynamics remain unclear in the supine position in chronic SCI.

In conclusion, passive ankle exercise was found to increase venous flow velocity in chronic SCI resulting in promoting venous return and eliminating stasis, but those with chronic SCI had a lower (roughly two-thirds) venous flow velocity than $\mathrm{AB}$. We considered that significant muscle atrophy and decreased blood distribution may have contributed to the smaller venous flow velocity in chronic SCI. Future studies involving combined measurement of muscle blood distribution would be helpful.

Conflict of interest

The authors declare no conflict of interest.

\section{ACKNOWLEDGMENTS}

The authors wish to thank the participants and the staff of the Gifu Central Hospital and the Nagoya City Sports Center for the Physically Handicapped for their assistance and support in this study.

\section{REFERENCES}

1) Merli GJ, Herbison GJ, Ditunno JF, et al.: Deep vein thrombosis: prophylaxis in acute spinal cord injured patients. Arch Phys Med Rehabil, 1988, 69: 661-664. [Medline]

2) Rossi EC, Green D, Rosen JS, et al.: Sequential changes in factor VIII and platelets preceding deep vein thrombosis in patients with spinal cord injury. Br J Haematol, 1980, 45: 143-151. [Medline] [CrossRef]

3) Sugimoto Y, Ito Y, Tomioka M, et al.: Deep venous thrombosis in patients with acute cervical spinal cord injury in a Japanese population: assessment with Doppler ultrasonography. J Orthop Sci, 2009, 14: 374-376. [Medline] [CrossRef]

4) Virchow R: Gesammelte abhandlungen zur wissenschaftlichen medizin. Meidinger Sohn Frankfurt, 1856, 219-226.

5) Kim SW, Charallel JT, Park KW, et al.: Prevalence of deep venous thrombosis in patients with chronic spinal cord injury. Arch Phys Med Rehabil, 1994, 75: 965-968. [Medline]

6) Dennis M, Sandercock PA, Reid J, et al. CLOTS Trials Collaboration: Effectiveness of thigh-length graduated compression stockings to reduce the risk of deep vein thrombosis after stroke (CLOTS trial 1): a multicentre, randomised controlled trial. Lancet, 2009, 373: 1958-1965. [Medline] [CrossRef]

7) McNally MA, Cooke EA, Mollan RA: The effect of active movement of the foot on venous blood flow after total hip replacement. J Bone Joint Surg Am, 1997, 79: 1198-1201. [Medline] [CrossRef]

8) Kwon OY, Jung DY, Kim Y, et al.: Effects of ankle exercise combined with deep breathing on blood flow velocity in the femoral vein. Aust J Physiother, 2003, 49: 253-258. [Medline] [CrossRef]

9) Sochart DH, Hardinge K: The relationship of foot and ankle movements to venous return in the lower limb. J Bone Joint Surg Br, 1999, 81: 700-704. [Medline] 
[CrossRef]

10) Lamb GC, Tomski MA, Kaufman J, et al.: Is chronic spinal cord injury associated with increased risk of venous thromboembolism? J Am Paraplegia Soc, 1993, 16: 153-156. [Medline] [CrossRef]

11) McCully KK, Mulcahy TK, Ryan TE, et al.: Skeletal muscle metabolism in individuals with spinal cord injury. J Appl Physiol 1985, 2011, 111: 143-148. [Medline] [CrossRef]

12) Stein PD, Matta F, Akkad MW, et al.: Effect of graduated compression stockings on venous blood velocity in supine resting hospitalized patients. Clin Appl Thromb Hemost, 2014, 20: 693-697. [Medline] [CrossRef] 